IOSR Journal of Pharmacy

e-ISSN: 2250-3013, p-ISSN: 2319-4219, www.iosrphr.org

Volume 2 Issue 6 ||| Nov-Dec. 2012 ||| PP.34-44

\title{
Solid Lipid Nanoparticle: A Review
}

\author{
Ramteke K.H ${ }^{1}$, Joshi S.A ${ }^{2}$, Dhole S.N ${ }^{3}$. \\ Department of Pharmaceutics, Modern College of Pharmacy (for Ladies), \\ Moshi, Pune 412105 Maharashtra, India
}

\begin{abstract}
Solid lipid nanoparticles are at the forefront of the rapidly developing field of nanotechnology with several potential applications in drug delivery, clinical medicine and research as well as in othervaried sciences.

Solid lipid nanoparticle (SLN) dispersions have been proposed as a new type of colloidal drug carrier system suitable for intravenous administration. The system consists of spherical solid lipid particles in the nanometer ranges, which are dispersed in water or in aqueous surfactant solution. It is identical to an oil-in-water emulsion for parenteral nutrition but the liquid lipid (oil) of the emulsion has been replaced by a solid lipid, i.e., yielding Solid Lipid Nanoparticles. Different production methods which are suitable for large scale production and applications of solid lipid nanoparticles are described. Appropriate analytical techniques for characterization of solid lipid nanoparticles like photon correlation spectroscopy, scanning electron microscopy, differential scanning calorimetry are highlighted. Aspects of solid lipid nanoparticles route of administration and their biodistribution are also incorporated. If appropriately investigated, solid lipid nanoparticles may open new vistas in therapy of complex diseases.
\end{abstract}

Keywords - Solid lipid nanoparticles (SLN), colloidal drug carriers, homogenization.

\section{INTRODUCTION}

Solid lipid nanoparticles (SLN) introduced in 1991 represent an alternative carrier system totradition colloidal carriers such as emulsions, liposomes and polymeric micro and nanoparticles [1].Nanoparticles made from solid lipids are attracting major attention as novel colloidal drug carrier forintravenous applications as they have been proposed as an alternative particulate carrier system. The system consists of spherical solid lipid particles in the nanometer ranges, which are dispersed in water or in aqueous surfactant solution. Generally, they are made of solid hydrophobic core having a monolayer of phospholipids coating. The solid core contains the drug dissolved or dispersed in the solid high melting fat matrix. The hydrophobic chains of phospholipids are embedded in the fat matrix. They have potential to carry lipophilic or hydrophilic drugs or diagnostics [2].

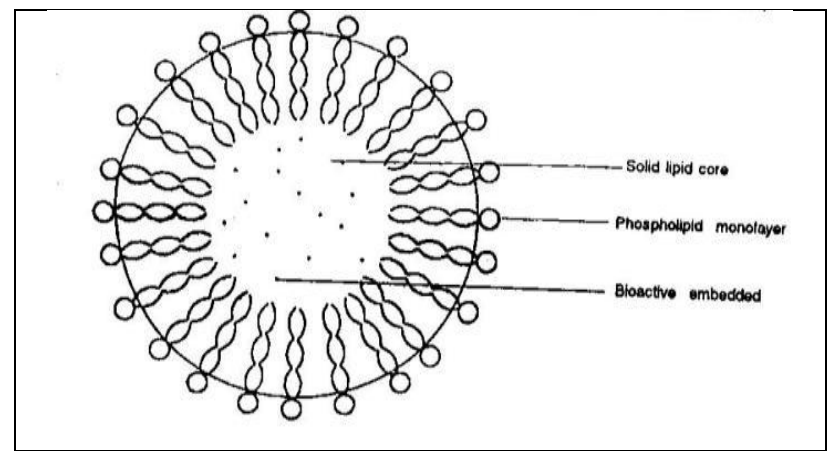

Fig. 1: Proposed structure of SLN.

SLN combine the advantages of polymeric nanoparticles, fat emulsion and liposomes but simultaneously avoid some of their disadvantages. They have many advantages such as good biocompatibility, non toxic, stable against coalescence, drug leakage, hydrolysis, biodegradable, physically stable and good carrier for lipophillic drugs [3]. There are major difference between lipid emulsion and liposomes. The basic structure of a lipid emulsion is a neutral lipophilic oil core surrounded by monolayer of amphiphilic lipid. In contrast, liposomes contain an outer bilayer of amphiphilic molecule such as phospholipid with an aqueous compartment inside [4]. 
Table no.1: Comparative properties of solid lipid nanoparticles, Polymeric nanoparticles,

\begin{tabular}{|c|c|c|c|c|c|}
\hline Sr. No. & Property & SLN & $\begin{array}{l}\text { Polymer } \\
\text { Nanoparticle } \\
\text { s }\end{array}$ & Liposomes & $\begin{array}{l}\text { Lipid } \\
\text { Emulsions }\end{array}$ \\
\hline 1 & Systemic toxicity & Low & $>$ or $=$ to SLN & Low & Low \\
\hline 2 & Cytotoxicity & Low & $>=$ to SLN & Low & Low \\
\hline 3 & $\begin{array}{l}\text { Residues from } \\
\text { organic solvents }\end{array}$ & No & Yes & $\begin{array}{l}\text { May or may } \\
\text { not }\end{array}$ & No \\
\hline 4 & $\begin{array}{ll}\text { Large } & \text { scale } \\
\text { production } & \end{array}$ & Yes & No & Yes & Yes \\
\hline 5 & $\begin{array}{l}\text { Sterilization by } \\
\text { autoclaving }\end{array}$ & Yes & No & No & Yes \\
\hline 6 & Sustained release & Yes & Yes & $<$ or $=$ to $\mathrm{SLN}$ & No \\
\hline 7 & Avoidance of RES & $\begin{array}{lr}\text { Depend } & \text { on } \\
\text { size } & \text { and } \\
\text { coating } & \\
\end{array}$ & No & Yes & Yes \\
\hline
\end{tabular}

\subsection{Advantages of SLN}

1. SLNs have better stability and ease of upgradability to production scale as compared to liposome.

2. In SLNs the lipid matrix is made from physiological lipid which decreases the danger of acute and chronic toxicity.

3. Very high long-term stability.

4. It is easy to manufacture than bipolymeric nanoparticles.

5. Better control over release kinetics of encapsulated compound.

6. SLNs can be enhancing the bioavailability of entrapped bioactive.

7. Chemical protection of labile incorporated compound.

8. Raw material which are to be required are same as that of emulsion.

9. Large scale production is possible.

10. High concentration of functional compound can be achieved.

11. Lyophilization possible.

\subsection{Disadvantage}

1. Poor drug loading capacity.

2. Drug expulsion after polymeric transition during storage.

3. Relatively high water content of the dispersions (70-99.9\%).

4. The low capacity to load hydrophilic drugs due to partitioning effects during the production process.

\section{METHODS OF PREPARATION OF SOLID LIPID NANOPARTICLES}

\subsubsection{Hot homogenization technique}

Melting of the lipid

Dissolution of the drug in the melted lipid

Mixing of the preheated dispersion medium and drug lipid melt

Premix using a stirrer to form a coarse pre-emulsion<smiles>[CH]</smiles>

High pressure homogenization at a temperature above the lipid melting point

$\mathrm{O} / \mathrm{W}$ - nano emulsion

Solidification of the nano-emulsion by cooling down to room Temperature to form SLN

The drug is dissolved or dispersed in melted solid lipid for SLN or in a mixture of liquid lipid (oil) and melted solid lipid for nanostructure lipid carrier.In the hot homogenization method the lipid melt containing drug is dispersed in a solution of the hot surfactant at same temperature $\left(5-10^{\circ} \mathrm{C}\right.$ above the melting point of the 
solid lipid or lipid blend) by high speed stirring. This pre-emulsion is then passed through a high pressure homogenizer adjusted to the same temperature generally applying three cycles at 500 bar or two cycles at 800 bars.

The hot homogenization technique can be used for lipophilic and insoluble drugs. As the exposure time to high temperature is relatively short, many heat sensitive drugs can be safely processed. The technique is not suitable for incorporation of hydrophilic drugs into SLN because higher portion of drugs in water during homogenization results in low entrapment efficiency $[5 ; 6]$.

\subsubsection{Cold homogenization technique}

Melting of the lipid

Dissolution /solubilization of the drug in the melted lipid

Solidification of the drug loaded lipid in liquid nitrogen or dry ice

Grinding in a powder mill (50-100 micrometer particles)

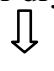

Dispersion of the lipid in the cold aqueous dispersion medium

Solid lipid nanoparticles

ᄀ

The first step of preparation is same as hot homogenization which includes dispersion or dissolving or solubilisation of the drug in the melted lipid. Then the drug lipid mixture is rapidlycooled either by means of liquid nitrogen or dry ice. The drug containing solid lipid is milled bymeans of mortar or ball mill to micron size (50-100 micron) and these microparticles aredispersed in chilled emulsifier solution yielding a pre-suspension.

Then this pre-suspension is subjected to high pressure homogenization at room or below room temperature, where thecavitation force is strong enough to break the microparticles to SLNs. This process avoids orminimizes the melting of lipid and therefore minimizing loss of hydrophilic drug to aqueousphase. Another method to minimize the loss of hydrophilic drug to aqueous phase is toreplace water with other media (e.g. oil or PEG 600) with low solubility for the drug. Incomparison to hot homogenization, in cold homogenization particle size and polydispersity indexare more. The cold homogenization only minimizes the thermal exposure of drug, but it does notavoid completely it due to melting of the lipid/drug mixture in the first step of preparation [7].

High pressure homogenization increases the temperature of the sample (e.g. $10-20^{\circ} \mathrm{C}$ for eachhomogenization cycle). In most of the cases, 3-5 homogenization cycles at 500-1500 bar aresufficient to prepare SLN. Increasing the number of homogenization cycle or thehomogenization pressure resulted in increase of particle size due to particle coalescence whichresulted from high kinetic energy of particles.

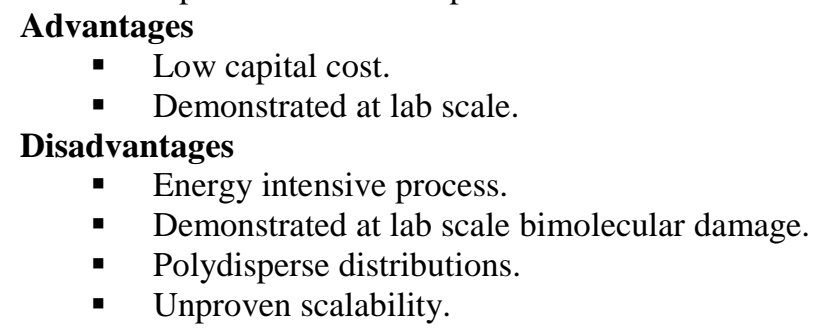

\subsection{Ultrasonication or High Speed Homogenization}

This ultrasonication technique is a dispersing technique, which was initially used for theproduction of solid lipid nanodispersion. Ultrasonication based on the mechanism ofcavitation. In first step, the drug was added to previously melt solid lipid. In second step, theheated aqueous phase (heated to same temperature) was added to the melted lipid and emulsifiedby probe sonication or by using high speed stirrer or aqueous phase added to lipid phase drop bydrop followed by magnetic stirring. The obtained pre-emulsion was ultrasonicated usingprobe sonicator with water bath $\left(\right.$ at $\left.0^{\circ} \mathrm{C}\right)$. In order to prevent recrystalization during the process, the production temperature kept at least $5^{\circ} \mathrm{C}$ above the lipid melting point. The obtainednanoemulsion $(\mathrm{o} / \mathrm{w})$ was filtered through a $0.45 \mu \mathrm{m}$ membrane in order to remove impuritiescarried in during ultrasonication [8]. Then they obtained SLN is stored at $4^{\circ} \mathrm{C}$. To increase thestability of the formulation, was lyophilized by a lyophilizer to obtain freeze-dried powder andsometime mannitol (5\%) was added into SLNs as cryoprotector. 
Advantages

- Reduced shear stress.

Disadvantages

- Potential metal contamination.

- Physical instability like particle growth upon storage.

\subsection{Solvent emulsification-evaporation technique}

In solvent emulsification-evaporation method, the lipophilic material and hydrophobic drug weredissolved in a water immiscible organic solvent (e.g. cyclohexane, dichloromethane, toluene,chloroform) and then that is emulsified in an aqueous phase using high speed homogenizer. To improve the efficiency of fine emulsification, the coarse emulsion wasimmediately passed through the microfluidizer. Thereafter, the organic solvent was evaporatedby mechanical stirring at room temperature and reduced pressure (e.g. rotary evaporator) leavinglipid precipitates of SLNs [9]. Here the mean particle size depends on theconcentration of lipid in organic phase. Very small particle size could be obtained with low lipidload (5\%) related to organic solvent.

Drug + lipid are dissolvedin $\mathrm{H} 2 \mathrm{O}$ immiscible solvent

Emulsification

In aqueous phase

$\mathrm{O} / \mathrm{w}$ emulsion

Solvent evaporation

at room temperature

and reduced pressure

SLN

Advantages

- Scalable.

- Mature technology.

- Continuous process.

- Commercially demonstrated.

Disadvantages

- Extremely energy intensive process.

- Polydisperse distributions.

- Bimolecular damage.

\subsection{Solvent emulsification-diffusion technique}

In solvent emulsification-diffusion technique, the solvent used (e.g. benzyl alcohol, butyl lactate,ethyl acetate, isopropyl acetate, methyl acetate) must be partially miscible with water and thistechnique can be carried out either in aqueous phase or in oil. Initially,both the solvent and water were mutually saturated in order to ensure the initial thermodynamicequilibrium of both liquid. When heating is required to solubilize the lipid, the saturationstep was performed at that temperature. Then the lipid and drug were dissolved in watersaturated solvent and this organic phase (internal phase) was emulsified with solvent saturatedaqueous solution containing stabilizer (dispersed phase) using mechanical stirrer. After theformation of $\mathrm{o} / \mathrm{w}$ emulsion, water (dilution medium) in typical ratio ranges from 1:5 to 1:10,were added to the system in order to allow solvent diffusion into the continuous phase, thusforming aggregation of the lipid in the nanoparticles. Herethe both the phase were maintain at same elevated temperature and the diffusion step was performed either at room temperature or at the temperature under which the lipid was dissolved. Throughout the process constant stirring was maintained. Finally, the diffused solvent waseliminated by vacuum distillation or lyophilization [7].

Solvent + Water are mutually saturated

Add drug and lipid emulsion

గु

$\mathrm{o} / \mathrm{w}$ emulsion+ Dilution media (water) in the ratio 1:5 - 1:10

Diffusion of solvent to continuous phase

SLN 


\title{
2.5 Micro emulsion based method
}

This method is based on the dilution of microemulsions. As micro-emulsions are two-phase systems composed of an inner and outer phase (e.g. o/w microemulsions). They are made by stirring an opticallytransparent mixture at $65-70^{\circ} \mathrm{C}$, which typically composed of a low melting fatty acid (e.g. stearic acid), an emulsifier (e.g. polysorbate 20), co-emulsifiers (e.g. butanol) and water. The hot microemulsion is dispersed in cold water $\left(2-3^{\circ} \mathrm{C}\right)$ under stirring. SLN dispersion can be used as granulation fluid for transferring in to solid product (tablets, pellets) by granulation process, but in case of low particle content too much of water needs to be removed. High-temperature gradients facilitate rapid lipid crystallization and prevent aggregation. Due to the dilution step; achievable lipid contents are considerably lower compared with the HPH based formulations [4].

Melting of lipid

Add aqueous solution of drug to melted lipid

Add Surfactant and co-surfactant at a temperature above the melting point of lipid

Formation of clear w/o microemulsion

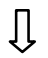

$\sqrt{2}$

Formed w/o microemulsion is added to a mixture of water, surfactant

and co-surfactant under mechanical stirring

Formation of suspension of lipid particles

Wash with dispersion medium by ultrafiltration system

Solid lipid nanoparticles

$\sqrt{3}$

\author{
Advantages \\ - Low mechanical energy input. \\ - Theoretical stability. \\ Disadvantages \\ - Extremely sensitive to change. \\ - Labor intensive formulation work. \\ - Low nanoparticle concentrations.
}

\subsection{Supercritical fluid technology}

This is a novel technique which recently applied for the production of SLNs. A fluid is qualifiedas supercritical when its pressure and temperature exceed their respective critical value. Above the critical temperature, it is not possible to liquefy a gas by increasing the pressure. The supercritical fluid has unique thermo-physical properties. As the pressure is raised, the density of the gas increases without significant increase in viscosity while the ability of the fluid to dissolve compounds also increases. A gas may have little to no ability to dissolve a compound under ambient condition can completely dissolve the compound under high pressure in supercritical range. Therefore, its solvation power is altered by careful control of changes in temperature and pressure. Many gases like, $\mathrm{CO}_{2}$, ammonia, ethane and $\mathrm{CH}_{2} \mathrm{FCF}_{3}$ were tried, but $\mathrm{CO}_{2}$ is the best option for SCF technique because, it is generally regarded as safe, easily accessible critical point $\left[31.5^{\circ} \mathrm{C}, 75.8\right.$ bar), does not causes the oxidation of drug material, leaves no traces behind after the process, is inexpensive, noninflammable, environmentally acceptable an easy to recycle or to dispose off. In the SCF phase or this technique generally use organic solvents (e.g. DMSO, DMFA) because they are fully miscible in $\mathrm{SCF}_{-} \mathrm{CO}_{2}$. This technology comprises several processes for nanoparticles production such as rapid expansion of supercritical solution (RESS), particles from gas saturated solution(PGSS), gas/supercritical antisolvent (GAS/SAS), aerosol solvent extraction solvent (ASES), solution enhanced dispersion by supercritical fluid (SEDS), supercritical fluid extraction of emulsions (SFEE). Mainly SAS and PGSS were used for SLN preparation [7].

\subsubsection{GAS/SAS}

In this process SCF acts as antisolvent for processing solid that are insoluble in SCF. It exploits the ability of SCF to dissolve in organic solvent and reduce the solvation power of solid in solution, thus causing the solid to precipitate. At first, the near critical or supercritical fluid was introduced in a vessel containing an organic solvent in which the solid material to be crystallized was dissolved which causes the intimate mixing of 
the fluid and liquid resulting in liquid expansion and particle precipitation. A clear disadvantage of this technique is the lack of control on the particle formation. A modification of SAS technique was used to prepare lysozyme spherical nanoparticles, which combines both the atomization and anti-solvent process, by using water/ethanol solution.

\subsubsection{PGSS}

In this process, the SCF was dissolved in liquid substrate, or a solution of substrate in solvent, or a suspension of substrate in solvent followed by a rapid depressurization of this mixture through a nozzle causing the formation of SLN. The great advantage of this process is that it produces particles of great variety of substance that need not be soluble in SCF-CO2. Limitations are, care must be taken for thermolabile solute and the final product may contain microparticles. Insulin nanoparticles are produced by this process, in which the solvent used, was DMSO and the lipid mixture (tristearin, phosphatidylcholine, dioctylsulfosuccinate) were atomized to produce insulin SLN $(<500 \mathrm{~nm})$.

\subsection{Double emulsion technique}

In double emulsion technique the drug (mainly hydrophilic drugs) was dissolved in aqueoussolution, and then was emulsified in melted lipid. This primary emulsion was stabilized byadding stabilizer (e.g. gelatin, poloxamer-407). Then this stabilized primary emulsion wasdispersed in aqueous phase containing hydrophilic emulsifier (e.g. PVA). Thereafter, the doubleemulsion was stirred and was isolated by filtration. Double emulsion technique avoidsthe necessity to melt the lipid for the preparation of peptide-loaded lipid nanoparticles and thesurface of the nanoparticles could be modified in order to sterically stabilize them by means ofthe incorporation of a lipid -PEG derivative. Sterical stabilization significantly improved theresistance of these colloidal systems in the gastrointestinal fluids [10]. This technique is mainlyused to encapsulate hydrophilic drug (peptides).

\subsection{Membrane contactor technique}

It is a novel technique to prepare the SLN. In membrane contactor technique the liquid phasewas pressed at a temperature above the melting point of the lipid through the membrane poresallowing theformation of small droplets as indicated in Figure 2. The aqueous phase was stirred continuouslyand circulates tangentially inside the membrane module, and sweeps away the droplets beingformed at the pore outlets. SLNs were formed by the cooling of the preparation at the roomtemperature. Here both the phases were placed in the thermostated bath to maintain the requiredtemperature and nitrogen was used to create the pressure for the liquid phase. The influence of various process parameters (aqueous phase cross flow velocity, the lipid phasepressure, aqueous and lipid phase temperature, lipid phase amount and membrane pore size) were studied. The membrane contactormethod is also used for the preparation of polymeric nanoparticles, by methods involving apolymerization of dispersed monomers (interfacial polymerization method) or a dispersion ofpreformed polymers (nanoprecipitation method). The advantages of this process of SLNpreparation using a membrane contactor are shown to be its facility of use, the control of theSLN size by an appropriate choice of process parameters and it's scaling up ability [7].

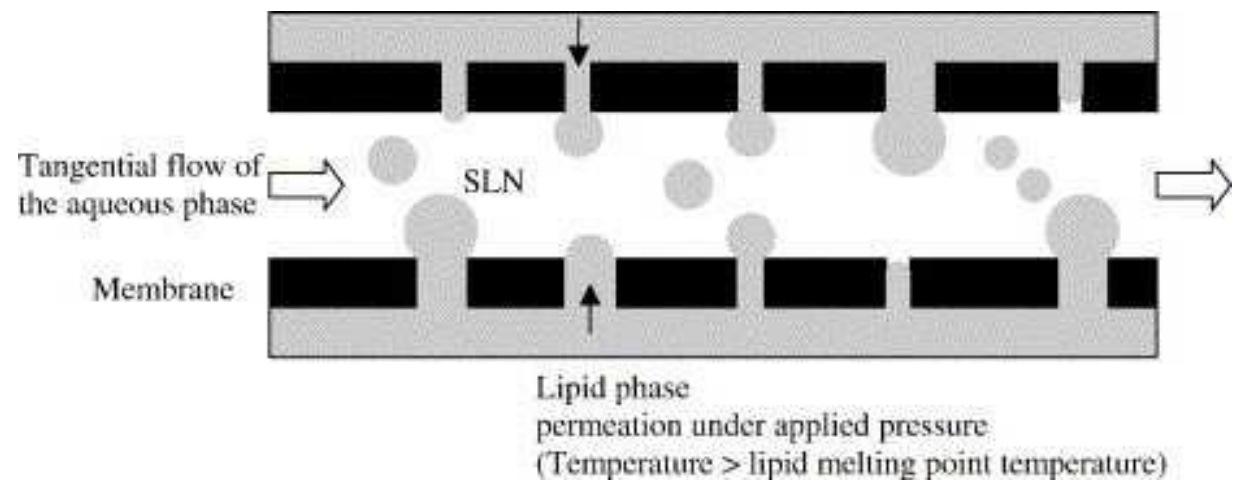

Fig. 2: Schematic drawing of the membrane contactor for the SLN preparation

\subsection{Solvent injection technique}

It is based onlipid precipitation from the dissolved lipid in solution. In this technique, the solid lipid wasdissolved in water-miscible solvent (e.g. ethanol, acetone, isopropanol) or a water-misciblesolvent mixture. Then this lipid solvent mixture was injected through an injection needle in tostirred aqueous phase with or without surfactant. The resulted dispersion was then filtered with afilter paper in order to remove any excess lipid. 
The presence of emulsifier within the aqueous phase helps to produce lipid droplets at the site ofinjection and stabilize SLN until solvent diffusion was complete by reducing the surface tensionbetween water and solvent resulting in solvent.

Advantages

- use of pharmacologically acceptable organic solvent

- easy handling

- Fast production process without technically sophisticated equipment.

\section{DRUG RELEASE FROM SLN}

There are mainly three drug incorporation models which describe the incorporation of drug into SLN [7].

1. Homogenous matrix model.

2. Drug enriched shell, core shell model.

3. Drug enriched core, core shell model.

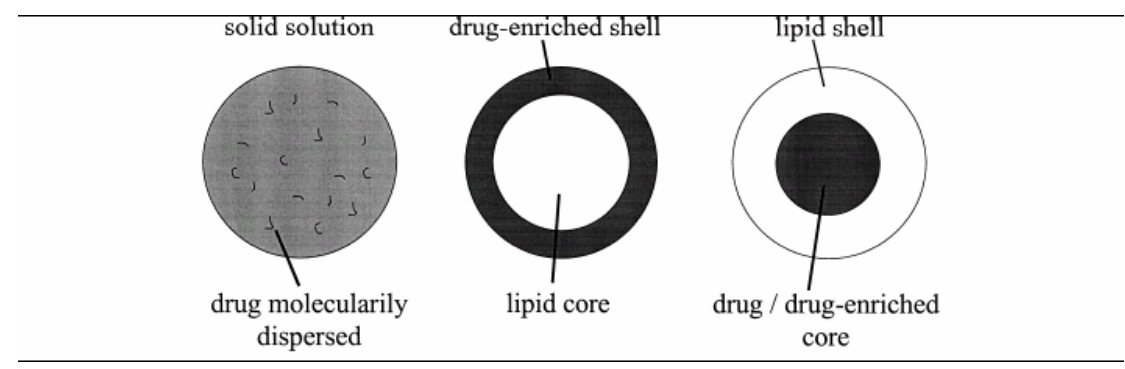

Fig. 3 Models of incorporation of active compounds into SLN:

(a) Homogeneous Matrix,

(b) Drug enriched shell with lipid core,

(c) Drug enriched core with lipid shell.

Homogenous matrix model or solid solution model with drug being present in amorphous clusters or molecularly dispersed is mainly obtained when incorporating highly lipophilic drugs into SLN with using hot homogenization technique or applying cold homogenization method or by avoiding potentially drug solubilizing surfactants. In the cold homogenization technique the drug (in molecularly dispersed form) is dispersed in bulk of melted lipid, then the mechanical force of high pressure homogenization leads to the breakdown of molecular form to nanoparticles and giving rise to homogenous matrix model as shown in Figure 3a. Etomidate SLN represents the homogenization matrix model.

The drug enriched shell with core shell model will be obtained when performing the production. During the production, the drug partitioned to water phase. Upon cooling, the lipid precipitates first, forming a practically drug free lipid core due to phase separation. At the same time, the drug re-partitions into the remaining liquid-lipid phase and drug concentration in the outer shell increasing gradually. Finally drug enriched shell crystallizes as depicted in Fig. 3b. The amount of drug partitioning to the aqueous phase will increases with the increase of solubility of drug in the aqueous phase. Mainly two factors, increasing temperature of the aqueous phase and increasing surfactant concentration, are increasing the saturation solubility of drug in water phase. Tetracaine SLN were prepared by hot HPH shows drug enriched shell model.

A drug enriched core obtained when dissolving a drug (e.g. prednisolone) in the lipid melts at or close to its saturation solubility. In this model, cooling of the formed nanoemulsion will lead to supersaturation of drug in melted lipid and it further leads drug precipitation prior to lipid precipitation. Further cooling will lead to precipitation of lipid surrounding the drug enriched core as a membrane as indicated in Figure 3c. Due to increased diffusional distance and hindering effect of surrounding solid lipid shell, the carrier system shows sustained release profile.

\section{CHARACTERIZATION OF SLNS}

\subsection{Measurement of particle size and zeta potential $[4 ; 11 ; 12]$}

Photon correlation spectroscopy (PCS) and laser diffraction (LD) are the most powerful techniques for routine measurements of particle size. PCS (also known as dynamic light scattering) measures the fluctuation of the intensity of the scattered light which is caused by particle movement. This method covers a size range from a few nanometers to about 3 microns. PCS is a good tool to characterize nanoparticles, but it is not able to detect larger micro particles. Electron Microscopy provides, in contrast to PCS and LD, direct information on the particle shape. The physical stability of optimized SLN dispersed is generally more than 12 months. ZP measurements allow predictions about the storage stability of colloidal dispersion. 


\subsubsection{PhotonCorrelation Spectroscopy (PCS)}

It is an established method which is based on dynamic scattering of laser light due to Brownian motion of particles in solution/suspension. This method is suitable for the measurement of particles in the range of $3 \mathrm{~nm}$ to $3 \mathrm{~mm}$.The PCS device consists of laser source, a sample cell (temperature controlled) and a detector.Photomultiplier is used as detector to detect the scattered light. The PCS diameter is based on the intensity of the light scattering from the particles.

\subsubsection{Electron Microscopy}

Electron Microscopy methods such as Scanning Electron Microscopy (SEM) and Transmission Electron Microscopy (TEM) are used to measure the overall shape and morphology of lipid nanoparticles. It permits the determination of particle size and distributions.SEM uses electrons transmitted from the surface of the sample while TEM uses electrons transmitted through the sample.

\subsubsection{Atomic Force Microscopy (AFM)}

It is an advanced microscopic technique which is applied as a new tool to image the original unchanged shape and surface properties of the particles. AFM measures the force acting between surface of the sample and the tip of the probe, when the probe is kept in close proximity to the sample which results in a spatial resolution of up to $0.01 \mathrm{~nm}$ for imaging.

\subsection{Determination of Incorporated Drug}

Amount of drug incorporated in SLNs influences the release characteristics hence it is very important to measure the amount of incorporated drug. The amount of drug encapsulated per unit wt. of nanoparticles is determined after separation of the free drug and solid lipids from the aqueous medium and this separation can be done by ultracentrifugation, centrifugation filtration or gel permeation chromatography. The drug can be assayed by standard analytical technique such as spectrophotometer, a spectroflurophotometry, HPLC or liquid scintillation counting.

\subsection{In vitro drug release}

\subsubsection{Dialysis tubing}

In vitro drug release could be achieved using dialysis tubing. The solid lipid nanoparticle dispersionis placed in pre washed dialysis tubing which can be hermetically sealed. The dialysis sac then dialyzedagainst a suitable dissolution medium at room temperature; the samples are withdrawn from the dissolutionmedium at suitable intervals, centrifuged and analyzed for the drug content using a suitable analyticalmethod.

\subsubsection{Reverse dialysis}

In this technique a number of small dialysis sacs containing $1 \mathrm{~mL}$ of dissolution medium are placedin SLN dispersion. The SLN's are then displaced into the medium.

\subsection{Rheology}

Rheological measurements of formulations can perform by Brookfield Viscometer, using a suitable spindle number. The viscosity depends on the dispersed lipid content. As the lipid content increases, the flow becomes non-Newtonian from Newtonian.

\subsection{Acoustic methods}

Another ensemble approach, acoustic spectroscopy, measures the attenuation of sound waves as a means of determining size through the fitting of physically relevant equations. In addition, the oscillating electric field generated by the movement of charged particles under the influence of acoustic energy can be detected to provide information on surface charge.

\subsection{Nuclear magnetic resonance (NMR)}

NMR can be used to determine both the size and the qualitative nature of nanoparticles. The selectivity afforded by chemical shift complements the sensitivity to molecular mobility to provide information on the physicochemical status of components within the nanoparticle.

\subsection{X-ray diffraction (powder X-ray diffraction) and differential scanning calorimetry (DSC)}

The geometric scattering of radiation from crystal planes within a solid allow the presence or absence of the former to be determined thus permitting the degree of crystallinity to be assessed.Another method that is a little different from its implementation with bulk materials, DSC can be used to determine the nature and speciation of crystallinity within nanoparticles through the measurement of glass and melting point temperatures and their associated enthalpies. 1 


\section{ROUTES OF ADMINISTRATION AND THEIR BIODISTRIBUTION}

The in vivo behavior of the SLN particles will mainly depend on the following points: Interactions of the SLN with the biological surroundings including: distribution processes (Adsorption of biological material on the particle surface and desorption of SLN components into tobiological surroundings) and enzymatic processes. Various administration routes are $[13 ; 14]$

\subsection{Parenteral administration}

Peptide and proteins drugs are usually available for parenteral use in the market. Since theirconventional oral administration is not possible due to enzymatic degradation in GI tract. Parenteralapplication of SLN reduces the possible side effects of drug incorporated with the increased bioavailability. These systems are very suitable for drug targeting.

\subsection{Oral administration}

Controlled release behavior of SLNs is reported to enable the bypass of gastric and intestinaldegradation of the encapsulated drug, and their possible uptake and transport through the intestinal mucosa.However, the assessment of the stability of colloidal carriers in GI fluids is essential in order to predict theirsuitability for oral administration.

\subsection{Rectal administration}

When rapid pharmacological effect is required, in some circumstances, parenteral or rectal

Administration is preferred. This route is used for pediatric patients due to easy application.

\subsection{Nasal administration}

Nasal route is preferred due to its fast absorption and rapid onset of drug action also avoidingdegradation of labile drugs in the GIT and insufficient transport across epithelial cell layers.

\subsection{Respiratory delivery}

Nebulisation of solid lipid particles carrying anti-tubercular drugs, anti-asthmatic drugs and anticancer was observed to be successful in improving drug bioavailability and reducing the dosing frequencyfor better management of pulmonary action.

\subsection{Ocular administration}

Biocompatibility and muco-adhesive properties of SLN improve their interaction with ocularmucosa and prolong corneal residence time of the drug, with the aim of ocular drug targeting.

\subsection{Topical administration}

SLN are very attractive colloidal carrier systems for skin applications due to their various desirableeffects on skin besides the characteristics of a colloidal carrier system. They are well suited for use ondamaged or inflamed skin because they are based on non-irritant and non-toxic lipids.

\section{APPLICATION}

\subsection{Oral SLNs in ant tubercular chemotherapy}

Ant tubercular drugs such as rifampicin, isonizide, pyrazinamide-loaded SLN systems, were able to decrease the dosing frequency andimprove patient compliance [15]. By using theemulsion solvent diffusion technique this antitubercular drug loaded solid lipidnanoparticles are prepared.

\subsection{SLNs for topical use}

SLNs used for topical application for various drugsuch as anticancer [16], vitamin-A [17], isotretinoin, flurbiprofen [18].Using glyceryl behenate, vitamineA-loaded nanoparticles can be prepared. Thismethod is useful for the improvement ofpenetration with sustained release. Theisotretinoin-loaded lipid nanoparticles wereformulated for topical delivery of drug.Productionof the flurbiprofen-loaded SLN gel for topicalapplication offer a potential advantage ofdelivering the drug directly to the site of action, which will produce higher tissue concentrations.

\section{SLNS AS COSMECEUTICALS}

The SLNs have been applied in the preparationof sunscreens and as an active carrier agent formolecular sunscreens and UV blockers. SLNand NLCs have proved to be controlled releaseinnovative occlusive topicals. Better localizationhas been achieved for vitamin A in upper layersof skin with glyceryl behenate SLNs compared toconventional formulations [19].

\subsection{SLNs as gene vector carrier}

SLN can be used in the gene vectorformulation. There are several recent reports ofSLN carrying genetic/peptide materials such asDNA, plasmid DNA and other nucleic acids [20]. The gene transfer was optimized byincorporation of a diametric HIV-1 HAT peptide(TAT 2) into SLN gene vector. The lipid nuclicacid nanoparticles were prepared from a liquidnanophase containing water and a watermiscible organic solvent where both lipid andDNA are separately dissolved by removing theorganic solvent, stable and homogeneously sizedlipid-nuclic acid nanoparticle $(70-100 \mathrm{~nm})$ wereformed. It's called genospheres. It is targetedspecific by insertion of an antibody-lipo polymerconjugated in the particle. 


\subsection{SLNs in breast cancer and lymph node metastases}

Mitoxantrone-loaded SLN local injections wereformulated to reduce the toxicity and improve thesafety and bioavailability of drug [21]. Efficacy ofdoxorubicin (Dox) has been reported to beenhanced by incorporation in SLNs.In themethodology the Dox was complexed withsoybean-oil-based anionic polymer anddispersed together with a lipid in water to formDox-loaded solid lipid nanoparticles. The systemhas enhanced its efficacy and reduced breastcancer cells.

\subsection{SLNs as a targeted carrier for anticancer drugto solid tumors}

SLNs have been reported to be useful as drugcarriers to treat neoplasm's [22]. Tumour targetinghas been achieved with SLNs loaded with drugs like methotrexate [23] and Camptothecin [24].Tamoxifen an anticancer drug is incorporated inSLN to prolong release of drug after i.v.

\subsection{Stealth nanoparticles}

These provide a novel and unique drug-delivery system they evade quick clearance by the immune system. Such nanoparticles can target specific cells. Stealth SLNs have been successfully tested in animal models with marker molecules and drugs. Antibody labelled stealth

Lipobodies have shown increased delivery to thetarget tissue in accessible sites [25].

\subsection{SLNs for potential agriculture application}

Essential oil extracted from Artemisiaarboreseens L when incorporated in SLN, wereable to reduce the rapid evaporation comparedwith emulsions and the systems have been usedin agriculture as a suitable carrier of ecologicallysafe pesticides [26].

\section{CONCLUSIONS}

SLN as colloidal drug carrier combines the advantage of polymeric nanoparticles, fat emulsions and liposome; due to various advantages, including feasibility of incorporation of lipophilic and hydrophilic drugs, improved physical stability, low cost, ease of scale-up, and manufacturing. SLNs are prepared by various advanced techniques. The site specific and sustained release effect of drug can better achieved by using SLNs. Nanoparticles have been used extensively for applications in drug discovery, drug delivery, and diagnostics and for many others in medical field. They are relatively novel drug delivery systems, having received primary attention from the early 1990s and future holds great promise for its systematic investigation and exploitation. We can expect many patented dosage forms in the form of SLNs in the future.

\section{REFERENCES}

[1]. P. Ekambaram, A. Abdul hasansathali K. Priyanka, solid lipid nanoparticles: a review Scientific Reviews Chemical Communications, 2(1), 2012, 80-102.

[2]. C. Shah, V. Shah, U. Upadhyay, solid lipid nanoparticles: a review current pharma research, 1(4), 2011, 351-368.

[3]. R. Cavalli, M. R. Gasco, P. Chetoni, S. Burgalassi, Solid lipid nanoparticles (SLN) as ocular delivery system for tobramycin. International Journal Pharm, 238, 2002, 241 - 245.

[4]. $\quad$ N. K. Jain, Controlled and Novel Drug Delivery 1st Edition, (CBS Publishers and Distributors1997) 3-28.

[5]. S.H. Gohla, A. Dingler, Scaling up feasibility of the production of solid lipid nanoparticles (SLN). Pharmazie, 56, 2001, 61-63.

[6]. C. Schwarz, W. Mehnert, J.S. Lucks, R.H. Müller, Solid lipid nanoparticles (SLN) for controlled drug delivery: I. Production, characterization and sterilization. Journal of Controlled Relelease, 30, 1994, 83-96.

[7]. P. Rabinarayan, S. Padilama, Production of Solid Lipid Nanoparticles-Drug Loading and Release Mechanism. Journal of Chemical and Pharmaceutical Research, 2(1), 2010,211-227.

[8]. T. Eldem, P. Speiser, A. Hincal, Optimization of spray-dried and congealed lipid microparticles and characterization of their surface morphology by scanning electron microscopy. Pharm Res. 8, 1991, 47-54.

[9]. B. Siekmann, K. Westesen. Investigations on solid lipid nanoparticles prepared by precipitation in o/w emulsions. European J. Pharm. Biopharm, 43, 1996, 104-109.

[10]. R. Cortesi, E. Esposito, G. Luca,C.Nastruzzi.Production of lipospheres as carriers for bioactive compounds. Biomaterials, 23, 2002, 2283-2294.

[11]. S. Pragati, S. Kuldeep, S. Ashok, Solid Lipid Nanoparticles: A Promising Drug Delivery Technology. International Journal of Pharmaceutical Sciences and Nanotechnology, 2(2), 2009, 509-516

[12]. S. N. Mistry, P. K. Patel, P. D. Bharadia, V. M. Pandya, D. A. Modi,Novel Drug Delivery System for Lipophilic Agents: Solid Lipid Nanoparticles. IJPI's Journal of Pharmaceutics and Cosmetology 1(4), 2011, 76-89.

[13]. S. P. Vyas and R. K. Khar, Controlled Drug Delivery - Concepts and Advances, 1st Edition, (VallabhPrakashan 2002) 38-50.

[14]. P. K. Gupta, J. K. Pandit, A. Kumar and P. Swaroop, S.gupta, T. Ph. Res.,3,2010117-138.

[15]. R.Pandey, S. Sharma, G.K. Khuller. Oral SLN Based antitubercular chemotherapy, Tuberculosis (Edinb) 85, 2005, 415-420.

[16]. H. Chen, X. Chang, D. Du, W. Liu, J. Liu, Podophyllotoxin- loaded solid lipid nanoparticles for epidermal targeting. Journal of Control Release 110, 2006, 296-306. 
[17]. V. Jenning, S. Gohla, Vitamin A-loaded solid lipid nanoparticles for topical use: drug release properties. Journal of Control Release, 66, 2000, 115-126.

[18]. M.C. Santos, W. Mehnert, M. Schaller, Drug targeting by solid lipid nanoparticles for dermal use. Journal Drug Target, 10, 2002, 489-495.

[19]. S.A. Wissing, R.H. Muller, Solid lipid nanoparticles (SLN) a novel carrier for U V blockers. Pharmazie 56, 2001, 783-786.

[20]. C. Rudolph, U. Schillinger, A. Ortiz, K. Tabatt, C. Plank, R.H. Muller,Application of novel Solid lipid nanoparticles (SLN) - gene vector formulations based on a diametric HIV-1 VAT - peptide in vitro and in vivo.Pharmaceutic Res, 21, 2004, 1662-1669.

[21]. B. Lu, S.B. Xiong, H. Yang, X.D. Yin, R.S. Chao, Solid lipid nanoparticles of mitoxantrone for local injection against breast cancer and its lymphnode metastases. European Journal of Pharm. Sciences, 28, 2006, 86-95.

[22]. V.S. Shenoy, I.K. Vijay, R.S. Murthy. Tumour targeting: Biological factors and formulation advances in injectable lipid nanoparticles. Journal Pharm Pharmacol, 57, 2005, 411-422.

[23]. K. Ruckmani, M. Sivakumar, P.A. Ganeshkumar, Methotrexate loaded solid lipid nanoparticles (SLN) for effective treatment of carcinoma. Journal Nano sciences Nanotechnology, 6, 2006, 2991-2995.

[24]. S.C.Yang,L.F.Lu, Y. Cai, J.B. Zhu, B.W. Liang, Body distribution in mice of intravenously injected camptothecin solid lipid nanoparticles and targeting effect on brain. Journal of Control Release, 59, 1999, 299-307.

[25]. Y. Wang, W. Wu, In situ evading of phagocytic uptake of stealth solid lipid nanoparticles by mouse peritoneal macrophages. Drug Delivery, 13, 2006, 189-192.

[26]. F. Lai, S.A. Wissing, R.H. Muller, A. M. FaddaArtemisia arborescensL essential oil-loaded solid lipid nanoparticles for potential agriculture application: Preparation and characterization. AAPS Pharm Sci Tech, 7, 2006, 21-27. 